\title{
Gastric Mesenchymal Myofibroblasts Maintain Stem Cell Activity and Proliferation of Murine Gastric Epithelium in Vitro
}

\author{
Takahito Katano, ${ }^{*}$ Akifumi Ootani, ${ }^{\dagger \dagger}$ Tsutomu Mizoshita, ${ }^{*}$ Satoshi Tanida, ${ }^{*}$ Hironobu Tsukamoto, ${ }^{*}$ Keiji Ozeki, ${ }^{*}$ \\ Hiromi Kataoka, ${ }^{*}$ and Takashi Joh*
}

\begin{abstract}
From the Department of Gastroenterology and Metabolism,* Nagoya City University Graduate School of Medical Sciences, Mizuho-ku, Nagoya; the Department of Gastroenterology, ${ }^{\dagger}$ Saiseikai Yahata General Hospital, Kitakyushu; and the Department of Internal Medicine, ${ }^{\ddagger}$ Faculty of Medicine, Saga University, Saga, Japan
\end{abstract}

\author{
Accepted for publication \\ November 7, 2014. \\ Address correspondence to \\ Tsutomu Mizoshita, M.D., \\ Ph.D., Department of Gastro- \\ enterology and Metabolism, \\ Nagoya City University Grad- \\ uate School of Medical \\ Sciences, 1 Kawasumi, \\ Mizuho-cho, Mizuho-ku, \\ Nagoya 467-8601, Japan. \\ E-mail: tmizoshi@med.nagoya- \\ cu.ac.jp.
}

\begin{abstract}
Stem cells are influenced by a microenvironmental niche that includes mesenchymal cells. We established a novel long-term method for primary mouse glandular stomach culture with mesenchymal myofibroblasts to investigate gastric epithelial-mesenchymal interactions. A gastric mesenchymal myofibroblast (GMF) cell line was established from mouse glandular stomach. Glandular stomach cells from neonatal mice and GMF cells were co-cultured in a collagen gel. Cultured stomach cells yielded expanding sphere-like structures. In the GMF co-culture system, the number and size of gastrospheres were increased compared with control cultures ( $P=0.009$ and 0.008 , respectively). Immunohistochemistry showed cells positive for human gastric mucin, HIK1083, and chromogranin A, indicating differentiation into surface mucous cells, mucous neck cells, and enteroendocrine cells, respectively. RNA in situ hybridization for Lgr5 showed $\mathrm{Lgr}^{+}$stem cells in the cultured gastrospheres. $\mathrm{Lgr}^{+}$cells were observed persistently in the epithelium of gastrospheres in the GMF co-culture system for 2 months. GMFs allowed the cultured gastric epithelium to maintain active proliferation similar to that seen in vivo. Real-time quantitative RT-PCR showed that Gas1 expression was higher in GMFs $(P=0.0445)$, and Hoxc8, Notch1, and Sox10 expressions were higher in intestinal mesenchymal myofibroblasts $(P=0.0003,0.0143$, and 0.0488 , respectively). We show the potential role of GMFs in sustaining $\mathrm{Lgr} 5^{+}$stem cell activity and affecting normal gastric epithelial differentiation and proliferation. (Am J Pathol 2015, 185: 798-807; http:// dx.doi.org/10.1016/j.ajpath.2014.11.007)
\end{abstract}

The homeostasis of the gastrointestinal mucosa is maintained by a variety of mechanisms. Epithelial cells of the gastric mucosa are organized in vertical flask-shaped structures called glands, which are composed of several epithelial cell types, including surface mucous (foveolar) cells, mucous neck cells, parietal cells, zymogenic (chief) cells, and enteroendocrine cells. ${ }^{1,2}$ The vertical unit of the gastric mucosa is composed of the pit, isthmus, neck, and base. ${ }^{1,2}$ The glands are surrounded by supporting stromal cells. Myofibroblasts in the scant mesenchyme between gland units are proposed to regulate stem cell activity. ${ }^{3}$

Stem cells generally are influenced by a microenvironmental niche, which typically is composed of epithelial and mesenchymal cells and extracellular substrates. ${ }^{4}$ The importance of epithelial-mesenchymal interactions has been shown in the development of various organs. ${ }^{5,6}$ To date, several factors including sonic hedgehog, transforming growth factor $\beta$, fibroblast growth factors, Wnt, hepatocyte growth factor, and bone morphogenetic proteins have been postulated to mediate epithelial-mesenchymal interactions. However, in the stomach, the mechanism of these interactions has not yet been elucidated fully. $^{7-14}$

In vitro analysis of gastric epithelial growth and differentiation has been hampered by a lack of appropriate culture

Supported by a Grant-in-Aid (Kiban C, 26460945) from the Ministry of Education, Culture, Sports, Science and Technology of Japan.

Disclosures: None declared. 
Table 1 Forty-Six Target Genes Used for Real-Time RT-PCR of Cultured Myofibroblasts

\begin{tabular}{|c|c|c|}
\hline Gene accession & Gene symbol & Gene description \\
\hline Mm01353100_m1 & Barx1 & BarH-like homeobox 1 \\
\hline Mm00432087_m1 & Bmp4 & Bone morphogenetic protein 4 \\
\hline Mm00432102_m1 & Bmp7 & Bone morphogenetic protein 7 \\
\hline Mm00552900_m1 & Boc & Biregional cell adhesion molecule-related/down-regulated by oncogenes (Cdon) binding protein \\
\hline Mm00477680_m1 & Btrc & $\beta$-Transducin repeat-containing protein \\
\hline Mm00491185_m1 & Cdon & Cell adhesion molecule-related/down-regulated by oncogenes \\
\hline Mm00433275_m1 & Fgf10 & Fibroblast growth factor 10 \\
\hline Mm00438917_m1 & Fgf4 & Fibroblast growth factor 4 \\
\hline Mm00546194_s1 & Foxc2 & Forkhead box $\mathrm{C} 2$ \\
\hline Mm00515793_m1 & Foxf2 & Forkhead box F2 \\
\hline Mm00433409_s1 & $F z d 7$ & Frizzled homolog 7 (Drosophila) \\
\hline Mm00469580_m1 & Hhip & Hedgehog-interacting protein \\
\hline Mm04213381_s1 & Hoxa5 & Homeobox A5 \\
\hline Mm00439369_m1 & Hoxc8 & Homeobox C8 \\
\hline Mm00439613_m1 & Ihh & Indian hedgehog \\
\hline Mm00496902_m1 & Jag1 & Jagged 1 \\
\hline Mm00550265_m1 & Lef1 & Lymphoid-enhancer binding factor 1 \\
\hline Mm00442942_m1 & Lifr & Leukemia inhibitory factor receptor \\
\hline Mm00464608_m1 & Lrp1 & Low-density lipoprotein receptor-related protein 1 \\
\hline Mm02384862_g1 & Nanog & Nanog homeobox \\
\hline Mm00476081_m1 & $N k \times 3-2$ & NK3 homeobox 2 \\
\hline Mm00435249_m1 & Notch1 & Notch 1 \\
\hline Mm01300162_m1 & Sox10 & SRY-box containing gene 10 \\
\hline Mm00448840_m1 & Soxg & SRY-box containing gene 9 \\
\hline Mm01219775_m1 & Stat3 & Signal transducer and activator of transcription 3 \\
\hline Mm00489385_m1 & Sufu & Suppressor of fused homolog (Drosophila) \\
\hline Mm00437328_m1 & Wnt11 & Wingless-related MMTV integration site 11 \\
\hline Mm00437337_m1 & Wnt3a & Wingless-related MMTV integration site $3 \mathrm{~A}$ \\
\hline Mm00437347_m1 & Wnt5a & Wingless-related MMTV integration site $5 \mathrm{~A}$ \\
\hline Mm00460518_m1 & Wnt9a & Wingless-type MMTV integration site $9 \mathrm{~A}$ \\
\hline Mm00656094_m1 & Zic1 & Zinc finger protein of the cerebellum 1 \\
\hline Mm01226725_g1 & Zic2 & Zinc finger protein of the cerebellum 2 \\
\hline
\end{tabular}

MMTV, mouse mammary tumor virus.

systems that recapitulate the in vivo environment. Several researchers have put a great deal of effort into generating longterm cultures of primary gastric epithelium, but the maintenance of gastric epithelial viability for more than 14 days is difficult, and the three-dimensional (3D) architecture of the gastric glands is not preserved. ${ }^{15-19}$ Therefore, current studies of gastric stem cells and their niche largely rely on the use of cell lines or studies in genetically manipulated mice, which are expensive and time consuming. ${ }^{20}$
Here, we describe a novel long-term method for culturing primary mouse glandular stomach cells to observe the dynamic effects of mesenchymal myofibroblasts in the stem cell niche on the differentiation of gastric epithelium. By using our culture method, we examined the effect of gastric mesenchymal myofibroblasts (GMFs) on the growth of gastric epithelial cells and found that GMFs contribute to the long-term maintenance of stem cell activity and proliferation of the gastric epithelium in vitro. Then, we identified 


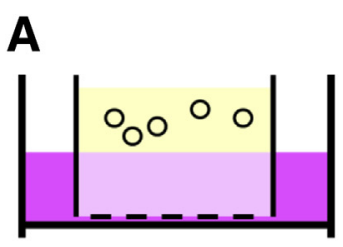

Control culture

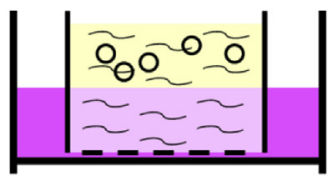

GMF co-culture
Collagen gel

Culture media

Gastric fragments

Gastric myofibroblasts

B

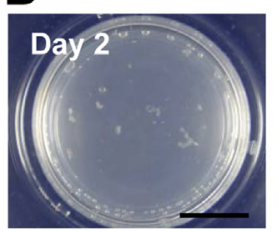

C

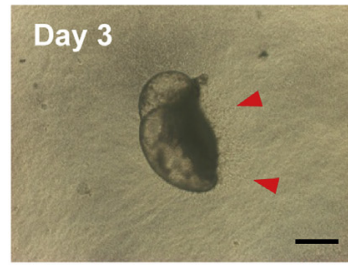

D
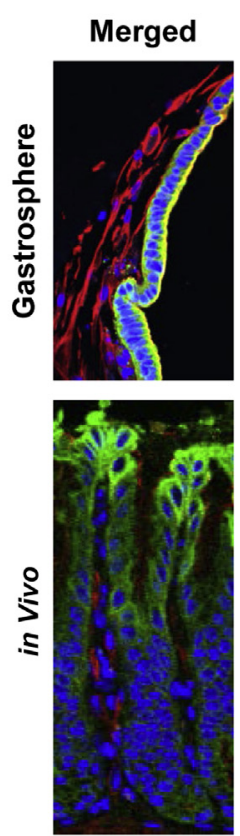
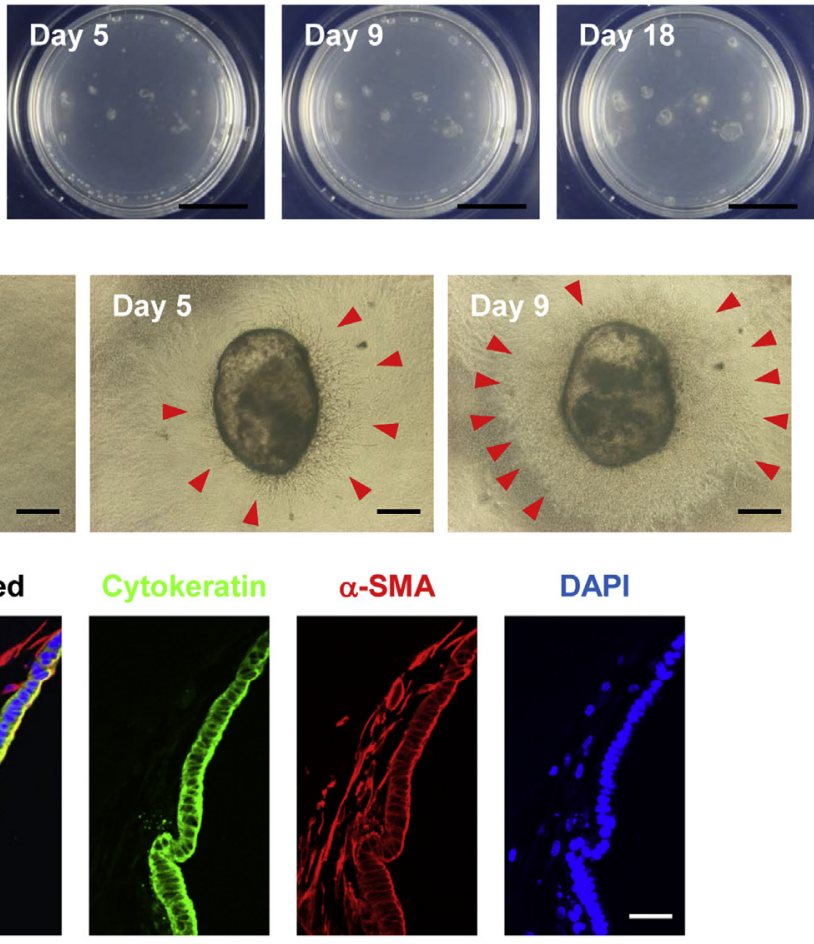

DAPI

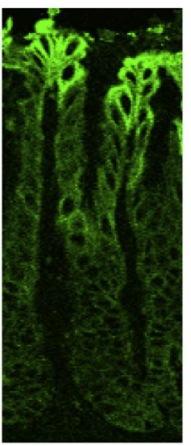

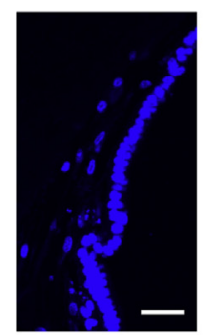
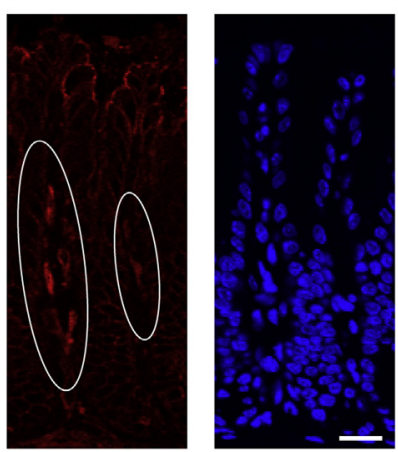

Figure 1 Long-term 3D culture of glandular stomach. A: Schematic diagrams of the control culture and GMF co-culture system. B: Time course analysis of the cultured stomach. Cultured glandular stomach within a collagen gel yields expanding cystic structures (gastrospheres). C: Phase-contrast microscopy. The gastrospheres grow in the presence of sufficient previously established outer growth of spindle-shaped myofibroblasts (red arrowheads). D: A gastrosphere on culture day 26 . Cultured gastrospheres mimic the in vivo environment of myofibroblasts and the gastric epithelium. Immunofluorescence showing a well-differentiated epithelium with an outer lining of $\alpha$-SMA-positive myofibroblasts. Gastric glands of an adult mouse show that, in vivo, $\alpha$-SMA -positive spindle cells (white circles) are present in the periglandular mesenchyme. Scale bars: $1 \mathrm{~cm}$ (B); $500 \mu \mathrm{m}$ (C); and $20 \mu \mathrm{m}$ (D). tissue-specific factors in GMFs and intestinal mesenchymal myofibroblasts (IMFs). This method will enable the study of gastric epithelial-mesenchymal interactions, as well as investigation of normal gastric epithelial growth and differentiation.

\section{Materials and Methods}

\section{D Primary Gastric Culture System}

The experimental design of this study was approved by the Animal Care Committee of the Nagoya City University Animal Research Institute. The animals were cared for in accordance with institutional guidelines, which complied with the instructions of the Health, Labor and Welfare Ministry concerning animal experiments.

We used the 3D culture system as previously reported. ${ }^{21,22}$ This system maintains cultured cells that are embedded in a collagen gel in an air-liquid interface environment. Cellmatrix type I-A (Nitta Gelatin, Inc., Osaka, Japan), Ham's F-12, and sterile reconstitution buffer $\left(2.2 \mathrm{~g} \mathrm{NaHCO}_{3}\right.$ in $100 \mathrm{~mL}$ of $0.05 \mathrm{~N} \mathrm{NaOH}$ and $200 \mathrm{mmol} / \mathrm{L}$ HEPES) were mixed at a ratio of $8: 1: 1$. This reconstituted collagen solution $(1.2 \mathrm{~mL})$ was poured into an inner 30-mm diameter dish (Millicell Culture Plate Inserts, PICM03050; Millicell-CM, Millipore, Billerica, MA). After 30 minutes at $37^{\circ} \mathrm{C}$, the collagen gel in 

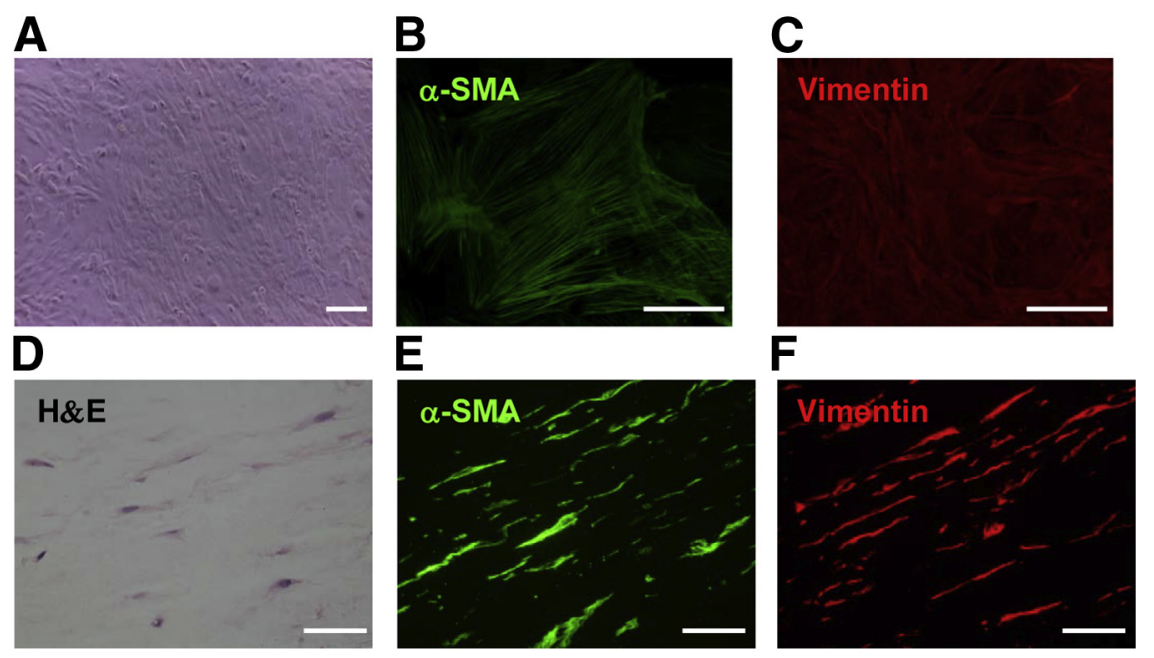

Figure 2 Establishment of a mouse GMF cell line. A: Phase-contrast microscopy. B and C: Immunofluorescence of GMF cells. $\alpha$-SMA (B) and vimentin (C) are expressed in GMF cells. D-F: Cultured GMF cells in the 3D culture system. D: Spindle-shaped cells grow in the collagen gel and are positive for $\alpha$-SMA (E) and vimentin (F). Scale bars: $50 \mu \mathrm{m}$. the inner dish had solidified, and the tissue was prepared. The glandular stomach was removed from postnatal day 2 C57BL/6J mice, placed on ice, and immediately washed in phosphate-buffered saline (PBS). Within 5 minutes, the washed tissue was minced into pieces smaller than $0.3 \mathrm{~mm}^{3}$ and mixed with the reconstituted collagen solution as described earlier. The tissue from one neonatal mouse was mixed in one dish. All work beginning from tissue removal until this step was performed on ice. The cell-containing collagen gel $(1.2 \mathrm{~mL})$ was poured onto the collagen gel in the inner dish, which was placed in a $60-\mathrm{mm}$ diameter outer dish. After the cell-containing gel solidified, $2.0 \mathrm{~mL}$ Ham's F12 supplemented with $20 \%$ fetal bovine serum and $50 \mu \mathrm{g} /$ $\mathrm{mL}$ gentamicin was poured into the outer dish, which was covered and incubated at $37^{\circ} \mathrm{C}$ in a humidified atmosphere of $5 \% \mathrm{CO}_{2}$ in air. Culture medium was replaced every 5 days. This culture system, which contained only mouse stomach tissue, was considered the control culture.

\section{Primary Culture of GMFs and the Co-Culture System}

The glandular stomach and the middle part of the small intestine were removed from postnatal day 2 C57BL/6J mice, placed on ice, and immediately immersed and washed in PBS. The washed tissue was minced into pieces smaller than $0.5 \mathrm{~mm}^{3}$ in the dishes (Becton Dickinson Bioscience, Franklin Lakes, NJ) and incubated in culture medium (Dulbecco's modified Eagle's medium and Ham's F12 in a 1:1 ratio, supplemented with $20 \%$ fetal bovine serum, $100 \mathrm{U} / \mathrm{mL}$ penicillin, $100 \mu \mathrm{g} / \mathrm{mL}$ streptomycin, and $0.25 \mu \mathrm{g} / \mathrm{mL}$ amphotericin B) at $37^{\circ} \mathrm{C}$ in a humidified atmosphere of $5 \% \mathrm{CO}_{2}$ in air. Minced pieces were grown attached to a dish. After 3 to 7 days, spindleshaped myofibroblasts had migrated from the minced tissue fragments and grown into confluent monolayers. These cells were passaged for subculturing and used as a source of GMFs and IMFs.

For the co-culture system, $5 \times 10^{5}$ GMFs were included under the collagen gel layer $(1.2 \mathrm{~mL})$ in the $3-\mathrm{cm}$ inner dish.
After the gel containing the GMFs solidified, the minced glandular stomach from one postnatal day 2 C57BL/6J mouse and another aliquot of $5 \times 10^{5}$ GMFs were mixed in $1.2 \mathrm{~mL}$ reconstituted collagen solution. The collagen gel containing minced tissue and the GMFs $(1.2 \mathrm{~mL})$ was poured onto the GMF-only collagen gel in the inner dish. Reconstituted collagen solution, culture medium, and culture conditions were the same as the control culture described earlier.

\section{Histology and Histochemistry}

Cultured cells in the collagen gel were fixed in $10 \%$ formalin, processed routinely, embedded in paraffin, sectioned, and stained with hematoxylin and eosin. To detect mucous substances, sections were stained with periodic acid-Schiff.

\section{Immunohistochemistry and Immunofluorescence}

Immunohistochemical and immunofluorescent staining was performed with antibodies against the following antigens: MUC5AC, MUC6, human gastric mucin (HGM), HIK1083, CD10 (1:100 each; Novocastra Laboratories, Newcastle upon Tyne, UK), MUC2 (1:100; Santa Cruz Biotechnology, Santa Cruz, CA), chromogranin A (1:1000; Yanaihara Institute, Inc., Fujinomiya, Japan), anti-proton pump/ $\mathrm{H}^{+}, \mathrm{K}^{+}$-adenosine triphosphatase (ATPase) (ready to use; MBL Co., Ltd., Nagoya, Japan), pepsinogen (1:10,000; Abcam, Tokyo, Japan), proliferating cell nuclear antigen (1:100; Dako, Glostrup, Denmark), Ki-67 (1:2000; Novocastra Laboratories), $\alpha$-smooth muscle actin ( $\alpha$-SMA) (1:200; Epitomics, Burlingame, CA), vimentin (1:250; Abcam), and cytokeratin (ready to use; Nichirei, Tokyo, Japan). The immunostaining procedures for paraffin sections were performed as described. ${ }^{23,24}$ For immunofluorescence, GMF cells grown on culture slides (Becton Dickinson Bioscience) were washed twice with PBS, fixed for 15 minutes in fresh 3\% paraformaldehyde diluted in PBS, permeabilized for 5 minutes in $0.5 \%$ Triton X-100 (Wako Pure Chemical Industries, Osaka, Japan) in PBS, 

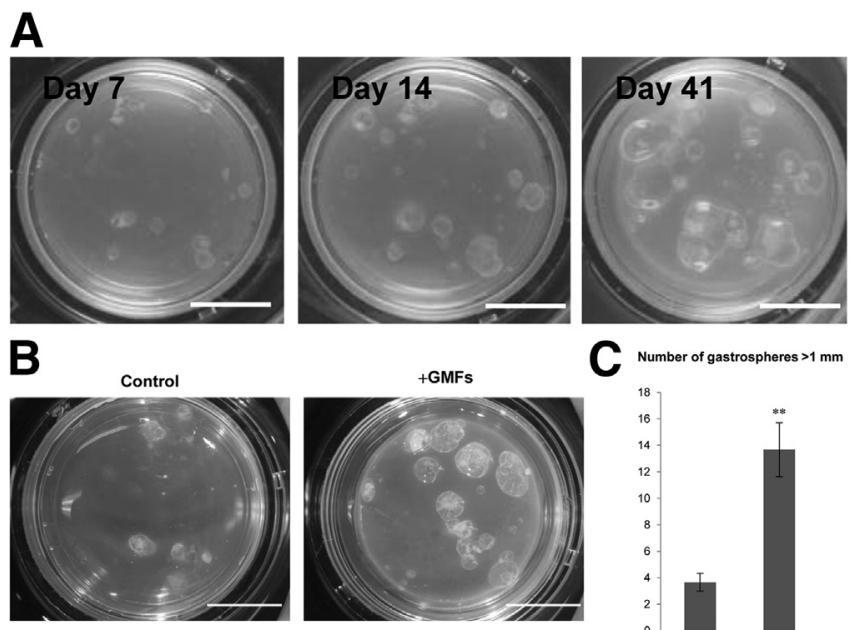

C

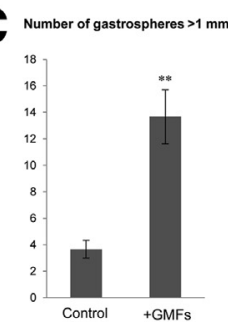

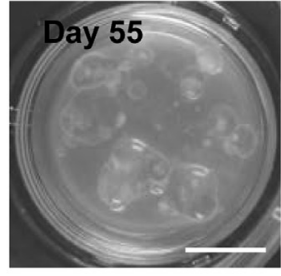

D

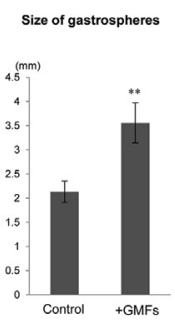

Figure 3 GMFs increase the number and size of gastrospheres in vitro. A: Time course analysis of cultured gastrospheres in the GMF co-culture system. B: Stereomicroscopy of cultured gastrospheres in the control culture and GMF co-culture system on day 20. C: The number of gastrospheres $>1 \mathrm{~mm}$ per culture dish was counted. D: The size of the three largest cultured gastrospheres per culture dish. Error bars indicate SEM. $n=3$ culture dishes. ${ }^{*} P<0.01$ versus control. Scale bars: $1 \mathrm{~cm}$. blocked for 60 minutes at room temperature in $10 \%$ normal goat serum, and then incubated in primary antibody for 60 minutes at room temperature. The slides were incubated with the corresponding secondary antibodies for 60 minutes at room temperature. ${ }^{23,24}$ The slides then were washed again and mounted with fluorescence mounting medium (Dako). As a positive control, normal mouse stomach and intestine were used for immunohistochemistry and immunofluorescence. The controls always showed positive results in a cell type-specific manner. Stained cells were observed with a confocal laser microscope (Nikon A1 Confocal System; Nikon Instech Co., Ltd., Tokyo, Japan), and data were analyzed using NIS element imaging software version 4.13 (Nikon Instech Co., Ltd.). Band-pass emission filters of 505 to $530 \mathrm{~nm}$ and $650 \mathrm{~nm}$ were used.

\section{RNA in Situ Hybridization}

In situ hybridization for Lgr5 was performed using the RNAscope FFPE assay kit (Advanced Cell Diagnostics, Inc., Hayward, CA) according to the manufacturer's instructions. Briefly, 4- to 5- $\mu \mathrm{m}$-thick formalin-fixed, paraffin-embedded tissue sections were pretreated with heat and protease digestion and then hybridized with a target probe for Lgr5. Horseradish-peroxidase-based amplification steps were performed. To detect target RNA, 3,3'-diaminobenzidine tetrahydrochloride was added. Positive staining was detected as brown punctate dots. The housekeeping gene peptidylprolyl isomerase $B$ was used as a positive control. The DapB gene was used as a negative control.

\section{Real-Time RT-PCR}

Muc5ac, Muc6, Pga5, PgC, Atp4a, Chga, and glyceraldehyde-3-phosphate dehydrogenase mRNA expression in gastrospheres cultured in the control system and the co-culture system was measured with real-time RT-PCR. In GMFs and IMFs, we compared mRNA expression levels of glyceraldehyde-3-phosphate dehydrogenase and
46 target genes related to morphogenesis, the hedgehog signaling pathway, bone morphogenetic protein, fibroblast growth factor, Notch, homeobox genes, and Wnt signaling (Table 1). Glyceraldehyde-3-phosphate dehydrogenase was chosen as an endogenous control to normalize the expression data. mRNA was reverse-transcribed into cDNA using a HighCapacity cDNA Reverse Transcription kit (Applied Biosystems, Tokyo, Japan) according to the manufacturer's instructions. TaqMan Gene Expression Assays for target genes and glyceraldehyde-3-phosphate dehydrogenase (Mm99999915_g1) were purchased from Applied Biosystems, and real-time quantitative RT-PCR analyses were performed in triplicate using an ABI 7500 Fast RealTime PCR system (Applied Biosystems) according to the manufacturer's recommendations. All data are presented as fold changes of the internal control.

\section{Cell Proliferation}

To calculate the percentage of $\mathrm{Ki}-67^{+}$cells, 500 cultured epithelial cells were counted, and the percentage of $\mathrm{Ki}-67^{+}$ nuclei was calculated. The data obtained from five to six independent experiments were analyzed with analysis of variance.

\section{Statistical Analysis}

The statistical significance of differences was determined using the Student's $t$-test or the Tukey-Kramer method. Differences were considered statistically significant at $P<0.05$. Data are expressed as means \pm SEM.

\section{Results}

Previously Established Growth of Gastric Myofibroblasts Is Essential for the Growth of Gastrospheres

We used our previously reported 3D culture system as the control culture (Figure 1A). ${ }^{22}$ Cultured minced gastric tissue 
fragments from neonatal mice yielded expanding cystic structures termed gastrospheres (Figure 1B) that were surrounded by outer spindle cells (Figure 1, C and D). The outer lining of spindle cells in the matrix was positive for the myofibroblast marker $\alpha$-SMA (Figure 1D). The previously established growth of gastric myofibroblasts was essential for the growth of gastrospheres. Gastric fragments without a sufficient number of outer myofibroblasts were not able to form gastrospheres. The placement of myofibroblasts in close proximity to the gastric epithelium in vitro mimicked the in vivo environment. $\alpha$-SMApositive spindle cells were present in the periglandular mesenchyme in vivo (Figure 1D).

\section{Establishment of a GMF Cell Line}

We established a mouse GMF cell line and used the cells at passages 4 to 10 in this study.

Cultured spindle cells were positive for the myofibroblast marker $\alpha$-SMA and vimentin (Figure 2, A-C), however, these cells were negative for cytokeratin, MUC5AC, HGM, MUC6, HIK1083, MUC2, CD10, chromogranin A, pepsinogen, and $\mathrm{H}^{+}, \mathrm{K}^{+}$-ATPase (data not shown). These results suggest that cultured cells had a similar expression pattern as gastric stromal myofibroblasts in vitro in the absence of epithelial cells. In our 3D culture system, cultured GMFs maintained similar immunohistochemical characteristics such as positive expression of $\alpha$-SMA and vimentin (Figure 2, D-F).

\section{Gastric Myofibroblasts Directly Increase the Number and the Size of Gastrospheres in Vitro}

Next, to investigate the effect of GMFs on the growth of gastrospheres, we used the co-culture system. In the GMF co-culture system, growth of gastrospheres was more stable over the long term (Figure 3, A and B). On culture day 20, we measured the size and calculated the number of gastrospheres that had grown to $>1 \mathrm{~mm}$ (Figure $3 \mathrm{~B}$ ). In the co-culture system and the control culture, the sizes of gastrospheres were $3.56 \pm 0.41 \mathrm{~mm}$ and $2.13 \pm 0.22 \mathrm{~mm}(P=0.008)$, respectively, and the numbers were $13.67 \pm 2.03$ and $3.67 \pm 0.67(P=0.009)$, respectively (Figure $3, \mathrm{C}$ and $\mathrm{D})$. Thus, GMFs significantly increased the growth and stability of gastrospheres.

\section{Gastric Myofibroblasts Induce Multilineage Differentiation of Gastric Epithelial Cells}

In both culture systems, the wall of the cultured gastrospheres consisted of a polarized epithelial monolayer with an apical, inner luminal surface, and a basal outer surface in close proximity to spindle-shaped cells and the collagen matrix. The cultured gastric epithelial cells showed a tall columnar shape, basally situated round nuclei, and clear cytoplasm that was filled with mucus (Figure 4A). The
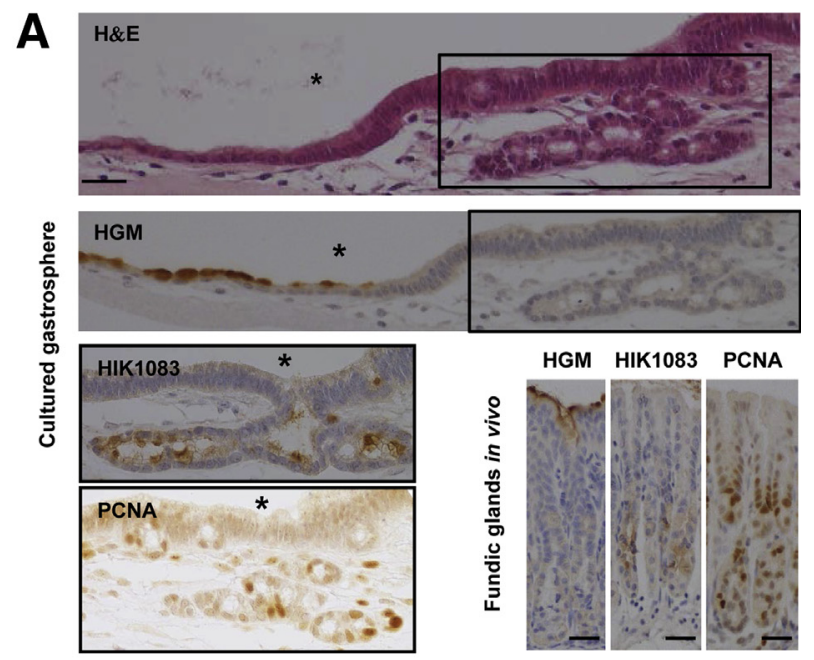

B

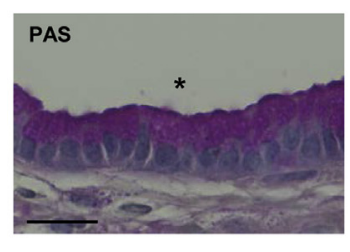

D

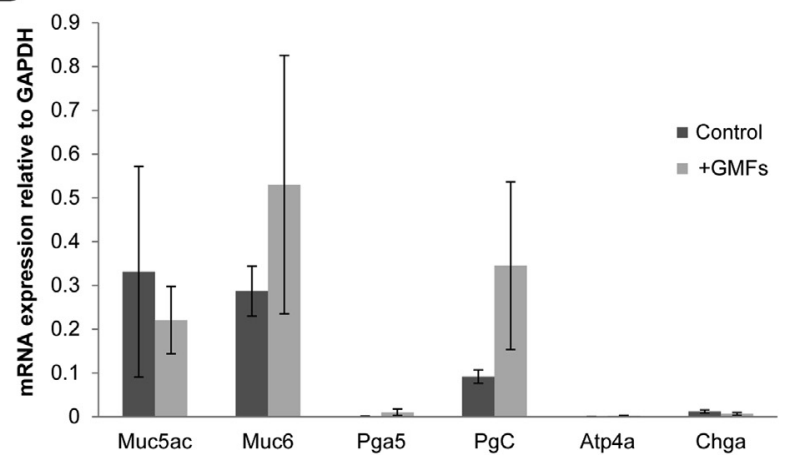

Figure 4 Differentiation and proliferation of gastrospheres. A: Boxes outline the same field of a gastrosphere on culture day 21 in the GMF co-culture system. The cultured gastric epithelial cells show a tall columnar shape, basally situated round nuclei, and clear cytoplasm. The gastric surface mucous cell marker HGM is expressed in the differentiated epithelial cells. The mucous neck cell marker HIK1083 is expressed in the pit-like structure. Proliferating cell nuclear antigen (PCNA) staining shows a proliferating zone in the gastrospheres that is similar to the isthmus of fundic glands in vivo. The foveolar differentiated epithelial cells show expression of HGM but not PCNA, mimicking the gastric surface mucosa in vivo. B: The intracytoplasmic mucus is stained strongly with periodic acid-Schiff (PAS). C: 0 n culture day 60, cells positive for chromogranin A are observed in the gastric epithelium (arrowheads). D: Real-time quantitative RT-PCR analyses for gastric epithelial markers in gastrospheres on culture day 20. Relative mRNA expression of Muc5ac, Muc6, Pga5, PgC, Atp4a, and Chga were not significantly different between gastrospheres in the control culture and those in the GMF co-culture system ( $P=0.638,0.521,0.337,0.315,0.235$, and 0.283 , respectively). Error bars indicate SEM. $n \geq 3$. Asterisks indicate the lumen of the sphere. Scale bars: $20 \mu \mathrm{m}$. GAPDH, glyceraldehyde-3-phosphate dehydrogenase.

intracytoplasmic mucus stained strongly with periodic acidSchiff (Figure 4B). In the control culture, HGM-positive epithelial cells and chromogranin A-positive cells were observed, indicating differentiation into gastric surface 
mucous cells and enteroendocrine cells, respectively. HIK1083-positive cells, indicating mucous neck cell differentiation, were scattered in the control culture on day 20 . However, the epithelial cells were negative for the parietal cell marker $\mathrm{H}^{+} / \mathrm{K}^{+}$-ATPase and the chief cell marker pepsinogen in the control culture on day 20. In the GMF co-culture system, in addition to HGM-positive cells, HIK1083-positive cells were observed in the pit-like structures of gastrospheres, and less than $1 \%$ of the epithelial cells were positive for the enteroendocrine cell marker chromogranin A (Figure 4, A-C). Even in the co-culture system, $\mathrm{H}^{+} / \mathrm{K}^{+}$-ATPase - and pepsinogen-positive cells were not observed with immunohistochemistry.

We next examined the effect of GMFs on the differentiation of the gastric epithelium as indicated by the expression of transcription factors. Muc5ac, Muc6, Pga5, PgC, Atp4a, and Chga expression was not significantly different between the co-culture system and control culture $(P=0.638,0.521$, $0.337,0.315,0.235$, and 0.283 , respectively) (Figure 4D).

\section{Maintenance of $\mathrm{Lgr}^{+}$Stem Cell Activity and Proliferation of Gastric Epithelium in Vitro}

We confirmed $\operatorname{Lgr} 5^{+}$cells using RNA in situ hybridization. $\mathrm{Lgr}^{+}$brown dots appeared at the base of crypts in the small intestines and colons of adult mice, and at the bottom of the pyloric glands in neonatal mice (Figure 5, A-D). In the gastrospheres on culture day $20, \mathrm{Lgr} 5^{+}$cells were detected in the epithelial layer (Figure 5E). On day 56, $\mathrm{Lgr}^{+}$cells were observed consistently in the epithelium of gastrospheres cultured in the GMF co-culture system (Figure 5E). In vitro and in vivo, $\mathrm{Lgr} 5^{+}$cells were observed only among epithelial cells, and not among mesenchymal myofibroblasts. We examined $\mathrm{Lgr}^{+}$epithelial cell populations in gastrospheres on day 20 in both culture systems. In the control culture system and the GMF co-culture, $\mathrm{Lgr}^{+}$epithelial cells were $7.15 \% \pm 1.12 \%$ and $9.96 \% \pm 3.61 \%(P=0.499)$, respectively, of the cells present (Figure $5 \mathrm{~F}$ ).

We next performed immunohistochemistry for Ki-67 to compare the proliferation ability of cultured epithelium in both culture systems (Figure 5G). The rate of Ki-67-positive cells in the GMF co-culture was higher than that in the control culture $(11.838 \% \pm 2.409 \%$ and $4.327 \% \pm 1.619 \%$, respectively; $P=0.032$ ). The rate of Ki-67-positive cells was not significantly different between gastrospheres in the GMF co-culture system and gastric glands in vivo $(11.838 \% \pm 2.409 \%$ and $15.473 \% \pm 0.314 \%$, respectively; $P=0.173$ ) (Figure 5G). These results suggest that GMFs allow maintenance of the cycle of gastric epithelial proliferation and differentiation, similar to that in vivo.

\section{GMFs and IMFs Express Tissue-Specific Factors}

To clarify the tissue-specific factors associated with the epithelial-mesenchymal interaction, we compared the expression levels of 46 genes between cultured GMFs and
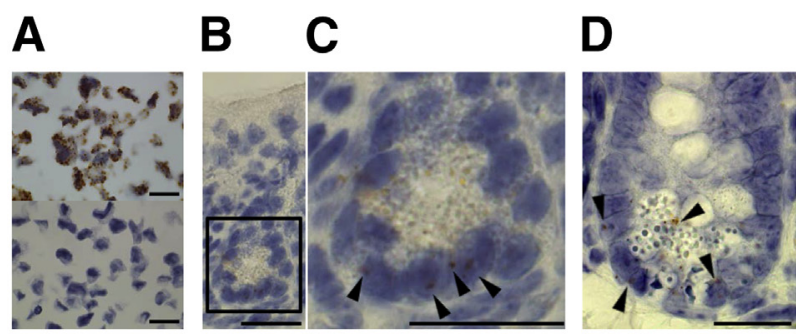

E
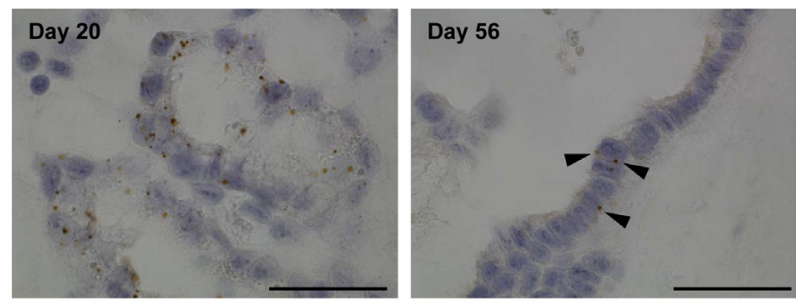

$\mathbf{F}$

G
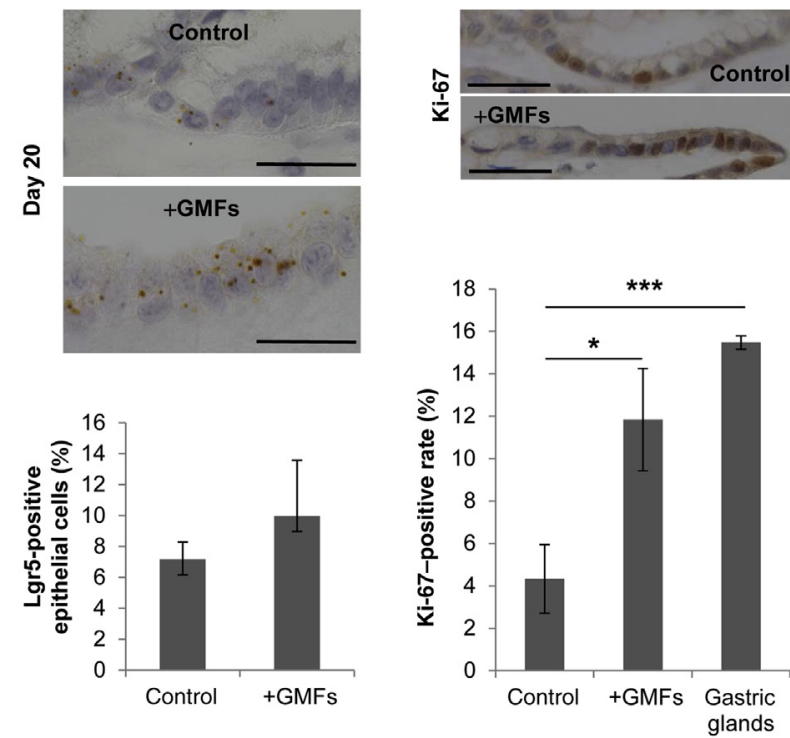

Figure 5 Maintenance of stem cell activity and proliferation of cultured gastrospheres. A-F: RNA in situ hybridization. A: Control slides of mouse 3T3 cell pellet. Top panel, the housekeeping gene peptidylprolyl isomerase $B$ served as a positive control. Positive staining is indicated by brown dots. Bottom panel, negative control using the DapB-negative control probe. $\mathbf{B}$ : RNA in situ hybridization for Lgr5 in pyloric glands of a neonatal day 2 mouse. C: Same field shown by the boxed area in panel B. Lgr5 ${ }^{+}$cells indicated by the brown dots (black arrowheads) are at the bottom of the pyloric glands of a neonatal day 2 mouse. D: RNA in situ hybridization for Lgr5 in the small intestine of an adult mouse. $\mathrm{Lgr}^{+}$cells are observed at the base of the crypt (black arrowheads). E: RNA in situ hybridization for Lgr5 in cultured gastrospheres on culture days 20 and 56. On culture day 20, $\mathrm{Lgr5}^{+}$cells were detected in the epithelial layer. $\mathrm{Lgr}^{+}$cells (black arrowheads) were observed consistently in the epithelium of gastrospheres on day 56. F: $\mathrm{Lgr5}^{+}$epithelial cell populations of gastrospheres on day 20 in both culture systems. In the control culture system and the GMF co-culture, $\mathrm{Lgr}^{+}$epithelial cells were $7.15 \% \pm 1.12 \%$ and $9.96 \% \pm 3.61 \%$ $(P=0.499)$, respectively, of the cells present. Error bars indicate SEM. G: Immunohistochemistry for Ki-67 in gastrospheres on culture day 20. The number of Ki-67-positive cells in gastrospheres in the control culture and GMF co-culture and in glandular stomach glands in vivo was counted. Error bars indicate SEM. $n=3(\mathbf{F}) ; n \geq 5(\mathbf{G}) .{ }^{*} P<0.05,{ }^{* *} P<0.001$ versus control. Scale bars: $20 \mu \mathrm{m}$. 

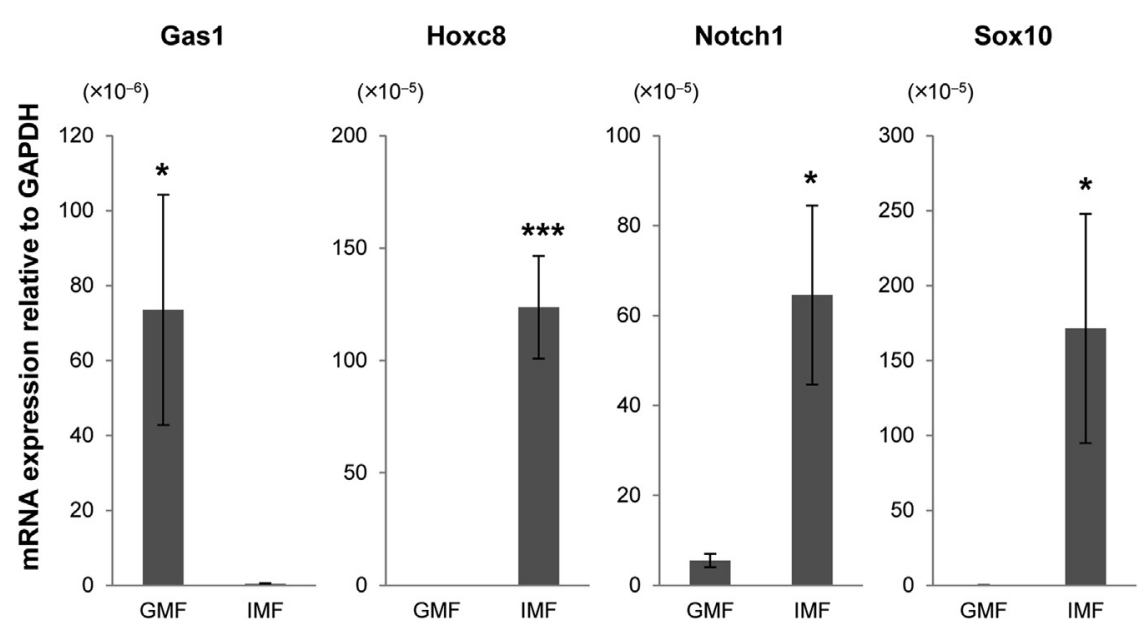

Figure 6 Real-time quantitative RT-PCR analyses of Gas1, Hoxc8, Notch1, and Sox10 in established GMF and IMF cell lines. Error bars indicate SEM. $n=5 .{ }^{*} P<0.05,{ }^{*} * * P<0.001$. GAPDH, glyceraldehyde-3-phosphate dehydrogenase.

small intestinal myofibroblasts using real-time RT-PCR. Among various genes associated with the hedgehog signaling pathway, Notch, bone morphogenetic protein, fibroblast growth factor, Wnt, and homeobox genes, the mRNA levels of four genes were significantly different between GMFs and IMFs (Figure 6). The expression of Gas1 in GMFs was significantly higher than in IMFs $(P=0.044)$. The expression of Notch1 and Sox 10 in IMFs was significantly higher than in GMFs $(P=0.014$ and 0.049 , respectively). Hoxc8 and Sox 10 were identified as tissue-specific factors expressed exclusively in IMFs $(P=0.0003)$.

\section{Discussion}

Long-Term 3D Primary Culture of Gastric Epithelial Cells

Here, we report a method for long-term culture of primary mouse glandular stomach cells with mesenchymal myofibroblasts as the stem cell niche. By using our previous 3D culture method, this method resulted in increased gastric epithelial stability and induced multilineage differentiation. In the past, several researchers have reported methods for primary gastric culture. ${ }^{15,16,18,19}$ In these reports of primary monolayer culture methods using human and animal specimens, cells could be cultured for only a few days to 2 weeks at most, and repeated passaging of these cultures was not possible. Therefore, current research regarding gastric stem cells, epithelial homeostasis, and gastric cancer relies heavily on the use of animal cell lines or genetically manipulated mouse models. ${ }^{20}$ Barker et al ${ }^{20}$ reported a culture system for generating and expanding long-lasting gastric organoids from sorted $\mathrm{Lgr}^{+}$cells. The most important difference is that without additional genetic manipulation, our new 3D culture method can reconstitute a similar in vivo environment of the stomach in vitro, including the stromal microenvironment. Although we have observed gastrosphere formation in adult mice, their viability was much lower than those from neonatal mice. Stable growth of gastrospheres from neonatal mice is easier to observe than growth from adult

mice. We believe that this depends on the stem cells present in adult mice, which are inferior to those in neonatal mice. Therefore, we used neonatal mice in this study.

\section{Gastric Stem Cells and Myofibroblasts as a Stem Cell Niche}

Myofibroblasts in the scant mesenchyme between gland units have been hypothesized to regulate stem cell activity.,25 Adult or somatic stem cells generally have limited functions outside their niche. ${ }^{4}$ Interactions between stem cells and the stem cell niche is the basis of tissue homeostasis. ${ }^{26-28}$ Here, we showed that dynamic, previously established growth of myofibroblasts was correlated highly with the growth of individual gastrospheres. Furthermore, additional tissue-specific myofibroblastic support in the collagen matrix allowed stable growth of gastrospheres and sustained gastric proliferation and differentiation for $\geq 50$ days.

Several investigators have reported candidate gastric stem cell markers. ${ }^{20,29-31}$ Lgr5 is expressed at the base of prospective corpus and pyloric glands in the neonatal stomach, and, using lineage tracing, $\mathrm{Lgr}^{+}$cells were shown to have multilineage potential. ${ }^{20}$ Because of the absence of a reliable monoclonal antibody to Lgr5, observing $\mathrm{Lgr}^{+}$cells in vitro has been difficult using methods other than genetically engineered mice. Here, we were able to specifically confirm the localization of $\mathrm{Lgr5}^{+}$cells in vitro using RNAscope. $\mathrm{Lgr}^{+}$cells were observed in the cultured gastric epithelium in the GMF co-culture system with RNA in situ hybridization on culture day 56. Thus, gastric myofibroblasts appeared to contribute to maintenance of somatic stem cell activity over the long term.

\section{Multilineage Differentiation of Gastric Epithelial Cells}

The mucous units of the pyloric antrum are populated by surface mucous cells that migrate outward and pyloric gland cells that migrate inward; their turnover times are approximately 3 and 1 to 60 days, respectively. ${ }^{32}$ Mucous neck cells migrate from the stem cell position in the gastric unit toward 
the base of the gastric glands. As these cells migrate downward, they transdifferentiate from mucous neck cells, which express Muc6, ${ }^{33}$ to zymogenic cells. Muc6 is identical to class III mucin, which is detected using immunohistochemistry with the specific antibody HIK1083. ${ }^{34}$ With histology and immunohistochemistry in the co-culture system, we confirmed HIK $1083^{+}$mucous neck cells and $\mathrm{HGM}^{+}$surface mucous cells forming pit-like structures, followed by formation of a monolayer of columnar epithelium. These results indicate that cultured gastric epithelium differentiates into both surface mucous cell lineage cells and mucous neck cell lineage cells in vitro. We did not observe cells positive for $\mathrm{H}^{+} / \mathrm{K}^{+}$-ATPase or pepsinogen here. We believe that these in vitro results depend on differences in turnover time, localization, and the number of parietal cells and zymogenic cells in vivo compared with the number of surface mucous cells, mucous neck cells, and enteroendocrine cells. The glandular stomach of neonatal day 2 mice is immature, and, therefore, few cells are available that can differentiate into parietal cells and zymogenic cells. The time required for differentiation of parietal cells and zymogenic cells is longer than surface mucous cells and mucous neck cells. Here, realtime RT-PCR showed mRNA expression of pepsinogen, and we suggest that cells were not sufficiently mature to produce the protein in vitro. In addition, immunohistochemistry for pepsinogen showed strong background staining, and, therefore, the primary antibody for pepsinogen that we used in this study may not have been appropriate for detecting zymogenic cells in vivo and in vitro. We believe that longer culture times may be necessary to observe differentiation of parietal cells and zymogenic cells in vitro, or additional specific factors may be necessary to induce differentiation of those cells in vitro. Our new GMF co-culture system allows stable longterm culture of gastric epithelium compared with the conventional method. By using our new method, we are now trying to culture the gastric epithelium for an extended period.

\section{Epithelial-Mesenchymal Interaction and Gastrointestinal Epithelial Phenotype}

Epithelial-mesenchymal interaction plays a central role in the morphogenesis, proliferation, and cytodifferentiation of various organs. ${ }^{5}$ Expression of Gas1, which is expressed in the developing mouse gastrointestinal tract, was significantly higher in GMFs than in IMFs. Inactivation of Gas 1 alone is sufficient to cause gastrointestinal defects related to reduced hedgehog signaling. ${ }^{35}$ During development, Gas1 and hedgehog signaling are correlated with stomach growth. However, the role of these signals in differentiation and proliferation in the adult stomach remains unclear. We propose that our co-culture system recapitulates the Gas1- and hedgehog-signal-dependent niche. With our 3D culture method, we were not able to distinguish between the interaction of GMFs with explanted myofibroblasts of minced tissue from the interaction with cultured myofibroblasts within the collagen gel. To evaluate the effects of soluble factors from GMFs, we suggest that placing GMFs in the bottom chamber, not within the collagen gel, will be useful in our 3D culture method.

In the stomach, Helicobacter pylori infection induces chronic gastritis, atrophy, and a shift from the gastric to the intestinal phenotype in both cancerous and non-neoplastic mucosa, and increases $\alpha$-SMA-positive myofibroblasts in the mesenchyme. ${ }^{36,37}$ Expression of $\mathrm{Cdx} 2$, an intestinalspecific gene, is important for intestinalization in both cancerous and non-neoplastic mucosa of the stomach, and $H$. pylori infection triggers ectopic $\mathrm{Cdx} 2$ expression in the stomach. ${ }^{36}$ However, the mechanism of transformation from the gastric to the intestinal phenotype remains unclear. Here, we showed the importance of support from gastric stromal myofibroblasts in gastric epithelial growth. We hypothesize that tissue-specific stromal myofibroblasts induce the gastric or intestinal epithelial phenotype. In stomach epithelial cells of the chicken embryo, a potential role for mesenchymal cells to influence the epithelial phenotype was reported. ${ }^{6}$ In our current study, Hoxc8 and Sox 10 were expressed specifically in IMFs. We suggest that tissue-specific myofibroblasts may induce each specific phenotype of the epithelium in the stomach and intestine. Our preliminary studies have suggested that tissue-specific myofibroblasts may induce each specific phenotype of the epithelium in the stomach and intestine and/or sustain the stem cell activity in the gastrointestinal tract. Further studies to elucidate the mechanism of the shift from the gastric to the intestinal phenotype currently are being performed in our laboratories.

\section{Conclusions}

In conclusion, we show the potential role of GMFs in affecting normal gastric epithelial differentiation and proliferation in our novel, long-term, in vitro 3D culture system. By analyzing the characteristic properties of myofibroblasts in the stomach and intestine, this culture method may be a valuable tool not only for studying normal gastrointestinal differentiation, but also for tissue engineering and regenerative medicine investigations.

\section{Acknowledgments}

We thank Yukimi Hashizume and Seizo Nagaya for expert technical assistance.

Conception and design of the work: T.K., T.M., S.T., and T.J.; analysis and interpretation of data: all authors; drafting the article: T.K., A.O., and T.M.; and final approval of the version to be published: all authors.

\section{References}

1. Karam SM, Leblond CP: Dynamics of epithelial cells in the corpus of the mouse stomach. I. Identification of proliferative cell types and pinpointing of the stem cell. Anat Rec 1993, 236:259-279 
2. Karam SM, Leblond CP: Dynamics of epithelial cells in the corpus of the mouse stomach. II. Outward migration of pit cells. Anat Rec 1993, 236:280-296

3. Mills JC, Shivdasani RA: Gastric epithelial stem cells. Gastroenterology 2011, 140:412-424

4. Scadden DT: The stem-cell niche as an entity of action. Nature 2006, 441:1075-1079

5. Gumbiner BM: Epithelial morphogenesis. Cell 1992, 69:385-387

6. Koike T, Yasugi S: In vitro analysis of mesenchymal influences on the differentiation of stomach epithelial cells of the chicken embryo. Differentiation 1999, 65:13-25

7. Kim JH, Huang Z, Mo R: Gli3 null mice display glandular overgrowth of the developing stomach. Dev Dyn 2005, 234:984-991

8. Suzuki H, Minegishi Y, Nomoto Y, Ota T, Masaoka T, van den Brink GR, Hibi T: Down-regulation of a morphogen (sonic hedgehog) gradient in the gastric epithelium of Helicobacter pylori-infected Mongolian gerbils. J Pathol 2005, 206:186-197

9. Halttunen T, Marttinen A, Rantala I, Kainulainen H, Maki M: Fibroblasts and transforming growth factor beta induce organization and differentiation of T84 human epithelial cells. Gastroenterology 1996, 111:1252-1262

10. Dignass AU, Tsunekawa S, Podolsky DK: Fibroblast growth factors modulate intestinal epithelial cell growth and migration. Gastroenterology 1994, 106:1254-1262

11. Korinek V, Barker N, Moerer P, van Donselaar E, Huls G, Peters PJ, Clevers H: Depletion of epithelial stem-cell compartments in the small intestine of mice lacking Tcf-4. Nat Genet 1998, 19:379-383

12. Montesano R, Matsumoto K, Nakamura T, Orci L: Identification of a fibroblast-derived epithelial morphogen as hepatocyte growth factor. Cell 1991, 67:901-908

13. Narita T, Saitoh K, Kameda T, Kuroiwa A, Mizutani M, Koike C, Iba H, Yasugi S: BMPs are necessary for stomach gland formation in the chicken embryo: a study using virally induced BMP-2 and Noggin expression. Development 2000, 127:981-988

14. Fukamachi H, Kato S, Asashima M, Ichinose M, Yuasa Y: Activin A regulates growth of gastro-intestinal epithelial cells by mediating epithelial-mesenchymal interaction. Dev Growth Differ 2013, 55: 786-791

15. Terano A, Ivey KJ, Stachura J, Sekhon S, Hosojima H, McKenzie WN Jr, Krause WJ, Wyche JH: Cell culture of rat gastric fundic mucosa. Gastroenterology 1982, 83:1280-1291

16. Sanders MJ, Amirian DA, Ayalon A, Soll AH: Regulation of pepsinogen release from canine chief cells in primary monolayer culture. Am J Physiol 1983, 245:G641-G646

17. Rattner DW, Ito S, Rutten MJ, Silen W: A rapid method for culturing guinea pig gastric mucous cell monolayers. In Vitro Cell Dev Biol 1985, 21:453-462

18. Chew CS: Parietal cell culture: new models and directions. Annu Rev Physiol 1994, 56:445-461

19. Ootani A, Toda S, Fujimoto K, Sugihara H: Foveolar differentiation of mouse gastric mucosa in vitro. Am J Pathol 2003, 162:1905-1912

20. Barker N, Huch M, Kujala P, van de Wetering M, Snippert HJ, van Es JH, Sato T, Stange DE, Begthel H, van den Born M, Danenberg E, van den Brink S, Korving J, Abo A, Peters PJ, Wright N, Poulsom R, Clevers H: Lgr5(+ve) stem cells drive self-renewal in the stomach and build long-lived gastric units in vitro. Cell Stem Cell 2010, 6:25-36

21. Ootani A, Li X, Sangiorgi E, Ho QT, Ueno H, Toda S, Sugihara H, Fujimoto K, Weissman IL, Capecchi MR, Kuo CJ: Sustained in vitro intestinal epithelial culture within a Wnt-dependent stem cell niche. Nat Med 2009, 15:701-706

22. Katano T, Ootani A, Mizoshita T, Tanida S, Tsukamoto H, Ozeki K, Ebi M, Mori Y, Kataoka H, Kamiya T, Toda S, Joh T: Establishment of a long-term three-dimensional primary culture of mouse glandular stomach epithelial cells within the stem cell niche. Biochem Biophys Res Commun 2013, 432:558-563

23. Mizoshita T, Tsukamoto $\mathrm{T}$, Nakanishi H, Inada K, Ogasawara N, Joh T, Itoh M, Yamamura Y, Tatematsu M: Expression of Cdx2 and the phenotype of advanced gastric cancers: relationship with prognosis. J Cancer Res Clin Oncol 2003, 129:727-734

24. Itoh K, Kataoka H, Sasaki M, Tanida S, Oshima T, Ogasawara N, Ohara H, Nakao H, Joh T: Bone morphogenetic protein 2 induced differentiation toward superficial epithelial cells in the gastric mucosa. J Gastroenterol 2006, 41:1064-1075

25. Morrison SJ, Spradling AC: Stem cells and niches: mechanisms that promote stem cell maintenance throughout life. Cell 2008, 132: 598-611

26. Schofield R: The relationship between the spleen colony-forming cell and the haemopoietic stem cell. Blood Cells 1978, 4:7-25

27. Xie T, Spradling AC: A niche maintaining germ line stem cells in the Drosophila ovary. Science 2000, 290:328-330

28. Xie T, Li L: Stem cells and their niche: an inseparable relationship. Development 2007, 134:2001-2006

29. Qiao XT, Ziel JW, McKimpson W, Madison BB, Todisco A, Merchant JL, Samuelson LC, Gumucio DL: Prospective identification of a multilineage progenitor in murine stomach epithelium. Gastroenterology 2007, 133:1989-1998

30. Quante M, Marrache F, Goldenring JR, Wang TC: TFF2 mRNA transcript expression marks a gland progenitor cell of the gastric oxyntic mucosa. Gastroenterology 2010, 139:2018-2027.e2

31. Stange DE, Koo BK, Huch M, Sibbel G, Basak O, Lyubimova A, Kujala P, Bartfeld S, Koster J, Geahlen JH, Peters PJ, van Es JH, van de Wetering M, Mills JC, Clevers H: Differentiated Troy+ chief cells act as reserve stem cells to generate all lineages of the stomach epithelium. Cell 2013, 155:357-368

32. Karam SM: Lineage commitment and maturation of epithelial cells in the gut. Front Biosci 1999, 4:D286-D298

33. Ho SB, Roberton AM, Shekels LL, Lyftogt CT, Niehans GA, Toribara NW: Expression cloning of gastric mucin complementary DNA and localization of mucin gene expression. Gastroenterology 1995, 109:735-747

34. Ishihara K, Kurihara M, Goso Y, Urata T, Ota H, Katsuyama T, Hotta K: Peripheral alpha-linked N-acetylglucosamine on the carbohydrate moiety of mucin derived from mammalian gastric gland mucous cells: epitope recognized by a newly characterized monoclonal antibody. Biochem J 1996, 318:409-416

35. Biau S, Jin S, Fan CM: Gastrointestinal defects of the Gas1 mutant involve dysregulated Hedgehog and Ret signaling. Biol Open 2013, 2: $144-155$

36. Tatematsu M, Tsukamoto T, Mizoshita T: Role of Helicobacter pylori in gastric carcinogenesis: the origin of gastric cancers and heterotopic proliferative glands in Mongolian gerbils. Helicobacter 2005, 10:97-106

37. Quante M, Tu SP, Tomita H, Gonda T, Wang SS, Takashi S, Baik GH, Shibata W, Diprete B, Betz KS, Friedman R, Varro A, Tycko B, Wang TC: Bone marrow-derived myofibroblasts contribute to the mesenchymal stem cell niche and promote tumor growth. Cancer Cell 2011, 19:257-272 